J. Austral Math. Soc. 19 (Series B), (1976), 432-451.

\title{
SIMILARITY SOLUTIONS OF THE STEADY STATE COSMIC-RAY EQUATION OF TRANSPORT
}

\author{
G. M. WEBB
}

(Received 26 Aprıl 1976)

(Revised 14 December 1976)

\begin{abstract}
Similarity solutions of the steady-state equation of transport for the distribution function $F_{0}$ of cosmic rays in the interplanetary region are obtained by the use of transformation groups. The solutions are derived in detail for a spherically-symmetric model of the interplanetary region with an effective radial diffusion coefficient $\kappa=\kappa_{0}(p) r^{b}$ with $r$ the heliocent ric radial distance, $p$ the particle momentum, $\kappa_{11}(p)$ an arbitary function of $p$, and the solar wind velocity is radial and of constant speed $V$. Solutions for which the similarity variable $\eta$ is a function of $r$ only are also derived; these are of particular importance when the $F_{0}$ is specified on a boundary of given radius. Non spherically-symmetric solutions can also be obtained by group methods and examples of such solutions are listed, without derivation, for the equation of transport incorporating the effects of anisotropic diffusion (diffusion coefficient $\kappa_{\|}$in the radial direction and $\kappa_{\perp}$ normal to it). The solutions are the most extensive steady-state analytic solutions yet obtained, and contain previous analytic solutions as special cases.
\end{abstract}

\section{Introduction}

The equation of transport for the propagation of cosmic-rays in interplanetary space including the physical processes of convection, diffusion and energy changes was formulated by Parker [17]. It was derived by a different method and a streaming equation added by Gleeson and Axford [11], and by Dolginov and Toptygin $[7,8]$. Since then many analyses of the consequences of this equation have been carried out, some by means of analytic solutions, but most using numerical solutions following Fisk [9].

In this paper the theory of Lie groups (Lie, [14]; Ovsjannikov, [16]; Matschat and Muller, [15]; Bluman, [2, 3, 4]; Bluman and Cole, [5, 6]) is used to obtain similarity solutions of the steady-state equation of transport. These 
solutions are the most general steady-state analytic solutions available, and they can be used to derive the previously reported analytic solutions of Parker $[17,18]$, Fisk and Axford [10], Toptygin [20], Webb and Gleeson [22, 23] and Gleeson and Webb [12].

The solutions are derived for a spherically-symmetric model of the interplanetary region, in which the effective radial diffusion coefficient $\kappa=\kappa_{0}(p) r^{b}$, where $p$ denotes particle momentum, $r$ is heliocentric distance, $b$ is a constant, and $\kappa_{0}(p)$ in an arbitrary function of momentum. The solar wind velocity is assumed to be radial and of constant speed $V$.

In section 2, the steady-state spherically symmetric equation of transport is expressed in a separable form, with separation variables $x$ and $t$, where $x$ is a function of $r$ and $p$, and $t$ is a function of $p$. A monoenergetic source term is included in the transport equation since it is intended (in a later paper) to derive the monoenergetic source or Green's function solution of Webb and Gleeson [22, 23] and of Toptygin [20] from the similarity solutions.

In section 3 , the infinitesimal transformations admitted by the separated transport equation are obtained, both for the case of a monoenergetic source term and for the homogeneous equation with zero source term.

In section 4, the infinitesimal transformations, determined in section 3 , and the group methods of Bluman and Cole [5, 6] are used to obtain spherically-symmetric similarity solutions of the equation of transport. In terms of the mean distribution function with respect to momentum $F_{0}(r, p)$, the solutions have the functional form

$$
F_{0}(r, p)=F(\eta) G[x(\eta, t), t],
$$

where $\eta(x, t)$ is one of the invariants of the group, known as a similarity variable, and $x=x(r, p)$ and $t=t(p)$ are the separation variables of section 2 . The dependence of $G$ on $\eta$ and $t$ is derived explicitly from invariance considerations. The function $F(\eta)$ is the solution of an ordinary differential equation obtaned by substituting the form (1.1) into the steady-state equation of transport for $F_{01}(r, p)$.

In the study of the propagation of cosmic-rays in the interplanetry region, solutions in which the distribution function is specified at one fixed heliocentric radius and appropriate boundary conditions are specified at another, are of particular interest. Since the similarity curves $\eta=$ constant, play the role of boundary curves in the general similarity form (1.1), solutions with boundaries $r=$ constant, are obtained by requiring that $\eta$ (or a limit of $\eta$ ) be solely a function of $r$. Examples of such solutions are obtained in section 5 , as particular cases of the general similarity solutions of section 4 .

In section 6 , further analytic similarity solutions of the equation of 
transport, including anisotropic diffusion, obtained by the group method are listed without derivation from Webb [21]. These solutions are for an idealised model with a radial interplanetary magnetic field, and for a radial solar wind velocity of constant speed $V$. The solutions are non spherically symmetric and depend on heliocentric position $(r, \theta, \phi)$ and momentum $p$, where $(r, \theta, \phi)$ are spherical polar coordinates centred on the sun with polar axis coincident with the sun's rotation axis. By appropriatc transformation of the diffusion coefficients parallel and perpendicular to the magnetic field, $\kappa_{\|}$and $\kappa_{1}$, the spherically-symmetric component of the latter solutions give the sphericallysymmetric solutions of section 4 .

Finally in section 7 , some comments are made about the scope and applicability of the present results and further papers to follow which extend this work are noted.

\section{Separation of the transport equation}

With a source of monoenergetic particles of momentum $p_{0}$, released at heliocentric radius $r_{0}$, the steady-state continuity equation for cosmic-rays propagating in the interplanetary medium is (Parker, [17]; Gleeson and Axford [11]; Dolginov and Toptygin [7, 8]; Jokipii and Parker [13])

$$
\begin{gathered}
\nabla \cdot\left(V F_{11}-\kappa \cdot \nabla F_{0}\right)-\frac{1}{3 p^{2}} \nabla \cdot V \frac{\partial}{\partial p}\left(p^{3} F_{0}\right) \\
=\frac{N}{16 \pi^{2} r_{0}^{2} p_{0}^{2}} \delta\left(r-r_{0}\right) \delta\left(p-p_{0}\right),
\end{gathered}
$$

where $F_{0}(r, p)$ is average distribution function with respect to momentum $p$. Here $\boldsymbol{V}$ is the solar wind velocity, $\boldsymbol{\kappa}$ is the diffusion tensor, $\delta(z)$ is a delta function of argument $z$, and $N$ is the number of particles released per unit time. The terms on the right represent the source.

For the spherically symmetric models considered here the diffusion tensor is replaced by an effective radial diffusion coefficient

$$
\kappa=\kappa_{0}(p) r^{b}
$$

where $\kappa_{0}(p)$ is an arbitrary function of momentum $p$, and the solar wind velocity is radial, and of constant speed $V$. With this model the transport equation (2.1) is

$$
\begin{gathered}
\kappa_{0}(p) r^{b+1} \frac{\partial^{2} F_{0}}{\partial r^{2}}+\left[(2+b) \kappa_{0}(p) r^{b}-V r\right] \frac{\partial F_{0}}{\partial r}+\frac{2 V p}{3} \frac{\partial F_{0}}{\partial p} \\
=\frac{-N}{16 \pi^{2} r_{0} p_{0}^{2}} \delta\left(r-r_{0}\right) \delta\left(p-p_{0}\right) .
\end{gathered}
$$


By introducing two new independent variables

$$
x=\left\{\begin{array}{lll}
2\left(r p^{3 / 2}\right)^{(1-b) / 2} /(1-b) & \text { if } & b \neq 1, \\
-\ln \left(2 r^{2} p^{3}\right) / 2 & \text { if } & b=1,
\end{array}\right.
$$

and

$$
t=-3 \int^{p} \kappa_{0}(z) z^{(1-3 b) / 2} d z / 2 V,
$$

the transport equation transforms into the separable forms

$$
M\left[F_{0}\right]=\frac{\partial F_{0}}{\partial t}-\left(\frac{a_{1}}{x}+a_{2}\right) \frac{\partial F_{0}}{\partial x}-\frac{\partial^{2} F_{0}}{\partial x^{2}}-Z \delta\left(x-x_{0}\right) \delta\left(t-t_{0}\right)=0,
$$

where

(i) if $b \neq 1$ and $\kappa=\kappa_{0}(p) r^{b}$,

$$
\begin{aligned}
& a_{1}=2 n+1, a_{2}=0, \\
& Z=3 N x_{0} /\left(32 \pi^{2} V p_{0}^{3} r_{0}^{2}|n+1|\right), \\
& n=(b+1) /(1-b),
\end{aligned}
$$

and

(ii) if $b=1$ so that $\kappa=\kappa_{0}(p) r$,

$$
\begin{aligned}
& a_{1}=0, a_{2}=-2, \\
& Z=3 N /\left(32 \pi^{2} V p_{0}^{3} r_{0}^{2}\right) .
\end{aligned}
$$

The forms (2.6) of the transport equation with parameter values (2.7) and (2.8) provide the basis of our subsequent study on the invariance group of (2.6) (section 3) and the similarity solutions of the spherically-symmetric transport equation (sections 4 and 5).

\section{Infinitesimal transformations admitted by the transport equation}

Denoting any solution of the transport equation for $F_{0}$ by $u(x, t)$, we now use the technique of Bluman and Cole [5] to determine the infinitesimal transformations

$$
\begin{aligned}
& x^{\prime}=x+\varepsilon X(x, t, u)+O\left(\varepsilon^{2}\right) \\
& t^{\prime}=t+\varepsilon T(x, t, u)+O\left(\varepsilon^{2}\right) \\
& u^{\prime}=u+\varepsilon U(x, t, u)+O\left(\varepsilon^{2}\right)
\end{aligned}
$$

admitted by the transport equation (2.6) which leave equation (2.6) invariant so that in terms of the differential operator $M$ of (2.6) we have

$$
M^{\prime}\left[u^{\prime}\right]=0 \quad \text { iff } \quad M[u]=0
$$


where $M^{\prime}\left[u^{\prime}\right]$ is obtained from $M[u]$ if $(x, t, u)$ is replaced by $\left(x^{\prime}, t^{\prime}, u^{\prime}\right)$. We obtain the infinitesimal generators $(X, T, U)$ of $(3.1)$ both for the case of a monoenergetic source term $-Z \delta\left(x-x_{0}\right) \delta\left(t-t_{0}\right)$ in equation (2.6) and for the homogeneous equation with zero source term.

The infinitesimal transformation of the delta function source term in (3.2) is obtained from

$$
\delta\left(x^{\prime}-x_{0}\right) \delta\left(t^{\prime}-t_{0}\right)=\delta\left(x-x_{0}\right) \delta\left(t-t_{0}\right) \frac{\partial(x, t)}{\partial\left(x^{\prime}, t^{\prime}\right)},
$$

where

$$
\frac{\partial(x, t)}{\partial\left(x^{\prime}, t^{\prime}\right)}=1-\varepsilon\left(X_{u} u_{x}+X_{x}+T_{t}+T_{u} l_{u}\right)+O\left(\varepsilon^{2}\right)
$$

is the Jacobian of the transformation between $(x, t)$ and $\left(x^{\prime}, t^{\prime}\right)$. The transformation of the $1 / x^{\prime}$ term in equation (3.2) is obtained by the Taylor series expansion

$$
\frac{1}{x^{\prime}}=\frac{1}{x}\left(1-\varepsilon \frac{X}{x}\right)+O\left(\varepsilon^{2}\right)
$$

From the partial derivative transformations of Bluman and Cole [5] and using the results (3.3)-(3.5) we have an expression for the transport equation operator on $u^{\prime}\left(x^{\prime}, t^{\prime}\right)$ in terms of the solution $u(x, t)$ :

$$
\begin{aligned}
M^{\prime}\left[u^{\prime}\right]= & M[u]+\varepsilon\left\{U_{t}-U_{x x}-\left(\frac{a_{1}}{x}+a_{2}\right) U_{x}+Z \delta\left(x-x_{0}\right) \delta\left(t-t_{0}\right)\right. \\
& +\left(X_{u} u_{x}+X_{x}+T_{t}+T_{u} u_{t}\right) \\
& +u_{t}\left[U_{u}-T_{t}+\left(\frac{a_{1}}{x}+a_{2}\right) T_{x}+T_{x x}\right] \\
& +u_{x}\left[-X_{t}+X_{x x}-2 U_{x u}-\left(\frac{a_{2}}{x}+a_{2}\right)\left(U_{u}-X_{x}\right)+\frac{a_{1} X}{x^{2}}\right] \\
& +u_{x}^{2}\left[2 X_{x u}-U_{u u}+\left(\frac{a_{1}}{x}+a_{2}\right) X_{u}\right] \\
& +u_{x} u_{t}\left[-X_{u}+2 T_{x u}+\left(\frac{a_{1}}{x}+a_{2}\right) T_{u}\right]-T_{u} u_{t}^{2}+X_{u u} u_{x}^{3} \\
& +T_{u u} u_{x}^{2} u_{t}+\left(2 X_{x}-U_{u}\right) u_{x x}+2 T_{x} u_{x x}+3 X_{u} u_{x} u_{x x} \\
& \left.+T_{u} u_{t} u_{x x}+2 T_{u} u_{x} u_{x x}\right\}+O\left(\varepsilon^{2}\right) .
\end{aligned}
$$

The 'classical method' of proceeding, described in Bluman and Cole [5] is to set the right-hand side of equation (3.6) proportional to $M[u]$. 
Thus the invariance condition (3.6) assumes the form,

$$
M^{\prime}\left[u^{\prime}\right]-M[u]=\varepsilon \lambda M[u]+O\left(\varepsilon^{2}\right),
$$

where $\lambda$ is at most a function of $x$ and $t$ (Ovsjannikov, [16], Chapter 6). Since $u$ is a solution of the transport equation (3.1) we have

$$
u_{x x}=-\left(\frac{a_{1}}{x}+a_{2}\right) u_{x}+u_{1}-Z \delta\left(x-x_{0}\right) \delta\left(t-t_{0}\right) .
$$

In the classical method we eliminate $u_{x x}$ from the invariance condition (3.6) or (3.7) by the substitution (3.8), and equate to zero terms with the same derivatives of $u$, i.e., the coefficients of $u_{t}, u_{x}, u_{x}^{2}, u_{x} u_{t}, u_{t}^{2}, u_{x}^{3}, u_{x}^{2} u_{t}, u_{x t}, u_{x} u_{x t}$, and the terms independent of the derivatives of $u$.

Successively equating to zero the coefficients of $u_{x} u_{x t}, u_{x} u_{t}$ and $u_{x}^{2}$ in equation (3.6) we find that

$$
T_{u}=0, \quad X_{u}=0, \quad U_{u u}=0,
$$

and consequently

$$
U=f(x, t) u+g(x, t), \quad X=X(x, t), \quad T=T(x, t) .
$$

Successively equating the coefficients of $u_{x t}, u_{t}, u_{x}$ and the remaining terms to zero,

$$
\begin{array}{r}
T_{x}=0, \text { and hence with }(3.10) T=T(t), \\
2 X_{x}-T^{\prime}(t)=0, \\
X_{t}-X_{x x}+2 f_{x}-\frac{a_{1} X}{x^{2}}+\left(\frac{a_{1}}{x}+a_{2}\right) X_{x}=0, \\
u\left[f_{x x}+\left(\frac{a_{1}}{x}+a_{2}\right) f_{x}-f_{t}\right]+g_{x x}+\left(\frac{a_{1}}{x}+a_{2}\right) g_{x}-g_{t} \\
-Z \delta\left(x-x_{0}\right) \delta\left(t-t_{0}\right)\left[T^{\prime}(t)+f(x, t)-X_{x}\right]=0 .
\end{array}
$$

From equation (3.14) we have

$$
\begin{gathered}
f_{x x}+\left(\frac{a_{1}}{x}+a_{2}\right) f_{x}-f_{t}=0, \\
g_{x x}+\left(\frac{a_{1}}{x}+a_{2}\right) g_{x}-g_{t}=0,
\end{gathered}
$$

and for a non-zero source term, with $Z \neq 0$,

$$
f\left(x_{0}, t_{0}\right)+T^{\prime}\left(t_{0}\right)-X_{x}\left(x_{0}, t_{0}\right)=0 .
$$

Note that $f(x, t)$ and $g(x, t)$ satisfy the homogeneous transport equation (2.6). 
The solutions of equations (3.10)-(3.17) for the infinitesimal generators $X, T$ and $U$ depend on the parameters $a_{1}$ and $a_{2}$ which in turn depend on the radial dependence of the diffusion coefficient according as $\kappa=\kappa_{0}(p) r^{b}, b \neq 1$ or $\kappa=\kappa_{0}(p) r$ (equations 2.6-2.8). The generators for these two cases are given below.

Case (i) $\kappa=\kappa_{0}(p) r^{b}, b \neq 1$

The parameters $a_{1}$ and $a_{2}$ for this case are given by equations (2.7), and the solutions of equations (3.10)-(3.17) for $X, T$ and $U$ are

$$
X=(\beta+\gamma t) x, \quad T=\gamma t^{2}+2 \beta t+\alpha, \quad U=u f+g,
$$

where

$$
f=-\frac{1}{4} \gamma x^{2}-(n+1) \gamma t+\delta
$$

$\alpha, \beta, \gamma, \delta$ are arbitrary parameters, and $g(x, t)$ is any solution of the homogeneous transport equation

$$
g_{x x}+\frac{2 n+1}{x} g_{x}=g_{i}
$$

Equations (3.18) give the infinitesimal generators for the homogeneous equation of transport (2.6). We now obtain the generators $(X, T, U)$ for the equation of transport with a monoenergetic source term.

For a source term in the transport equation (2.6) the source point $\left(x_{0}, t_{0}\right)$ is invariant under the infinitesimal transformation (3.1) and hence

$$
X\left(x_{0}, t_{0}\right)=T\left(t_{0}\right)=0 .
$$

Applying the source condition (3.17) and the results (3.19) for the invariance of the source point $\left(x_{0}, t_{0}\right)$, we find that the subgroup of the main group (3.18) admitted by the inhomogeneous equation has generators

$$
X=\gamma\left(t-t_{0}\right) x, \quad T=\gamma\left(t-t_{0}\right)^{2}, \quad U=u f+g,
$$

where

$$
f=-\frac{1}{4} \gamma\left(x^{2}-x_{0}^{2}\right)-(n+\dot{1}) \gamma\left(t-t_{0}\right)
$$

Case (ii) $\kappa=\kappa_{0}(p) r$

For this diffusion coefficient case, the parameters $a_{1}$ and $a_{2}$ occurring in the transport equation (2.6) are given in equations (2.8), and the solutions of the determining equations (3.10)-(3.17) for the infinitesimal generators $X, T$ and $U$ are 


$$
X=(\beta+\gamma t) x+k+\delta t, \quad T=\gamma t^{2}+2 \beta t+\alpha, \quad U=u f+g
$$

where

$$
f=-\frac{1}{4} \gamma x^{2}+\left(\beta+\gamma t-\frac{1}{2} \delta\right) x-\gamma t^{2}-\left(2 \beta+\frac{1}{2} \gamma-\delta\right) t+\lambda,
$$

$\alpha, \beta, \gamma, k, \lambda$ are arbitrary, but constant parameters, and $g(x, t)$ is any solution of the homogeneous transport equation

$$
g_{x x}-2 g_{x}=g_{1} .
$$

Equations (3.21) give the infinitesimal generators for the homogeneous equation of transport (2.6). Note that the generators $X$ and $T$ of equations (3.21a) are identical to the generators $X$ and $T$ obtained by Bluman and Cole [5] in their study of the one-dimensional heat equation.

The infinitesimal generators $X, T$ and $U$ for the equation of transport (2.6) with a monoenergetic source term $Z \delta\left(x-x_{0}\right) \delta\left(t-t_{0}\right)$ satisfy the invariant source condition (3.17) and the condition (3.19) corresponding to the invariance of the source point $\left(x_{0}, t_{0}\right)$. Thus the subgroup of the main group (3.21) admitted by the inhomogeneous equation of transport (2.6) has generators

$$
\begin{aligned}
& X=\beta\left(x-x_{01}\right)+\gamma\left(x t-x_{0} t_{0}\right)+\delta\left(t-t_{0}\right), \\
& T=\gamma\left(t^{2}-t_{0}^{2}\right)+2 \beta\left(t-t_{0}\right), \\
& U=u f+g,
\end{aligned}
$$

where

$$
\begin{aligned}
f= & -\frac{1}{4} \gamma\left(x^{2}-x_{0}^{2}\right)+\left(\beta-\frac{1}{2} \delta\right)\left(x-x_{0}\right)+\gamma\left(t x-t_{0} x_{0}\right) \\
& -\gamma\left(t^{2}-t_{0}^{2}\right)-\left(2 \beta+\frac{1}{2} \gamma-\delta\right)\left(t-t_{0}\right)-\left(\beta+\gamma t_{0}\right),
\end{aligned}
$$

and $g$ is a solution of the homogeneous transport equation (3.21c).

\section{Similarity solutions}

In this section the generators $(X, T, U)$ of the infinitesimal transformations obtained in section 3 , are used to derive similarity solutions of the cosmic-ray equation of transport (2.6) by the group methods of Bluman and Cole $[5,6]$. There are two cases to consider according as (i) $\kappa=\kappa_{0}(p) r^{b}, b \neq 1$ and (ii) $\kappa=\kappa_{\text {n }}(p) r$.

From Bluman and Cole $[5,6]$ the functional forms of the similarity solutions are obtained by integrating the group trajectories

$$
\frac{d x}{X}=\frac{d t}{T}=\frac{d u}{U} .
$$


In the present application the infinitesimal generators $(X, T, U)$ are given explicitly for the two clases in equations (3.18) and (3.21), and in each case are of the form

$$
X=X(x, t), \quad T=\gamma t^{2}+2 \beta t+\alpha, \quad U=u f(x, t)+g(x, t) .
$$

Here the solutions are obtained for generators with $g=0, \gamma \neq 0$, and for the two diffusion cocfficient cases (i) and (ii). Thus the group trajectories (4.1) may be written as

$$
\begin{aligned}
& \frac{d x}{d t}=\frac{X}{T}=A(x, t), \\
& \frac{d u}{d t}=\frac{U}{T}=\frac{u f}{T}=u C(x, t) .
\end{aligned}
$$

The solution of the trajectory equation (4.3) is of the form

$$
\eta(x, t)=\text { constant }=\eta_{c},
$$

where $\eta(x, t)$ is a similarity variable. Using the solution (4.5) to express $x$ in terms of $\eta_{c}$ and $t$ and substituting in the second trajectory equation (4.4), we obtain an ordinary differential equation in $u$ and $t$. Thus the solution of the second trajectory equation is of the form

where

$$
u=F\left(\eta_{c}\right) G\left[x\left(\eta_{c}, t\right), t\right],
$$

$$
G(x, t)=\exp \left(\int C\left[x\left(\eta_{c}, t\right), t\right] d t\right),
$$

and the constant of integration has been written as $F\left(\eta_{c}\right)$ with $F$ an arbitrary function of its argument.

The intersection of the surfaces $(4.5)$ and $(4.6)$ in $(x, t, u)$ space with $\eta_{c}$ constant is a curve which defines a group trajectory. The family of group trajectories obtained by varying the parameter $\eta_{c}$ generates the invariant solution surface or the functional form of the similarity solution. Thus the general similarity solution has the form

$$
u=F(\eta) G[x(\eta, t), t]
$$

where

$$
G[x(\eta, t), t]=\exp \left(\int C[x(\eta, t), t] d t\right),
$$

and $\eta(x, t)$ is given by equation (4.5). Note that the result (4.8) is the form of the similarity solution referred to in equation (1.1). Substitution of the form (4.8) into the transport equation (2.6) leads to an ordinary differential 
equation for $F(\eta)$, so that the similarity solution is completely determined. We now carry out the derivation of $G$ and $F$ for the particular cases of interest here.

Case (i) $\kappa=\kappa_{\mathrm{u}}(p) r^{b}, b \neq 1$

By setting

$$
t_{s}=-\beta / \gamma, \quad a_{s}=\left(\alpha \gamma-\beta^{2}\right) / \gamma^{2}, \quad \chi=[\delta+(n+1) \beta] / \gamma,
$$

in the generators (3.18), the trajectory (4.3) is

$$
\frac{d x}{d t}=A(x, t)=\frac{\left(t-t_{s}\right) x}{\left(t-t_{s}\right)^{2}+a_{s}}
$$

and the trajectory $(4.4)$ is

$$
\frac{1}{u} \frac{d u}{d t}=C(x, t)=\frac{-\frac{1}{4} x^{2}-(n+1)\left(t-t_{s}\right)+\chi}{\left(t-t_{s}\right)^{2}+a_{s}} .
$$

Following the general development of Bluman and Cole [5], integration of equation (4.11) yields the similarity variable

$$
\eta(x, t)=x /\left[\left(t-t_{s}\right)^{2}+a_{s}\right]^{\frac{1}{2}} .
$$

Then substituting

$$
x=\eta\left[\left(t-t_{s}\right)^{2}+a_{s}\right]^{\frac{1}{2}},
$$

from equation (4.13) in the trajectory equation (4.12), treating $\eta$ as a constant, and integrating yields the form of similarity solution

$$
u=F(\eta) \exp \left(-\frac{\eta^{2}\left(t-t_{s}\right)}{4}-\frac{n+1}{2} \ln \left[\left(t-t_{s}\right)^{2}+a_{s}\right]+\chi \int^{t-t_{s}} \frac{d y}{y^{2}+a_{s}}\right),
$$

analogous to the result (4.8). Note that the integral in the exponent is given explicitly by

$$
\int^{t-t_{s}} \frac{d y}{y^{2}+a_{s}}=\left\{\begin{array}{l}
\arctan \left[\left(t-t_{\mathrm{s}}\right) / a\right] / a \\
-1 /\left(t-t_{s}\right), \\
\ln \left[\left(t-t_{s}-b\right) /\left(t-t_{s}+b\right)\right] / 2 b
\end{array}\right.
$$

according as $a_{s}=a^{2}>0, a_{s}=0$, or $a_{s}=-b^{2}<0$, respectively.

The ordinary differential equation for $F(\eta)$ is now obtained by substituting the similarity form (4.15) into the homogeneous equation of transport (2.6). It is 


$$
\frac{d^{2} F}{d \eta^{2}}+\frac{2 n+1}{\eta} \frac{d F}{d \eta}+\left(\frac{\eta^{2} a_{s}}{4}-\chi\right) F(\eta)=0,
$$

where $n=(b+1) /(1-b)$ (equations 2.7).

The general solution of the differential equation (4.17) for $F(\eta)$ depends on the parameter $a_{s}$. For $a_{s} \neq 0$, the solution is

$$
\begin{aligned}
F(\eta)= & \exp \left(-\frac{\left(-a_{s}\right)^{\frac{1}{2}} \eta^{2}}{4}\right)\left[A M\left(\frac{1+n}{2}+\frac{\chi}{2\left(-a_{s}\right)^{\frac{1}{2}}}, n+1, \frac{\left(-a_{s}\right)^{\frac{1}{2}} \eta^{2}}{2}\right)\right. \\
& \left.+B U\left(\frac{1+n}{2}+\frac{\chi}{2\left(-a_{s}\right)^{\frac{1}{2}}}, n+1, \frac{\left(-a_{s}\right)^{\frac{1}{2}} \eta^{2}}{2}\right)\right],
\end{aligned}
$$

where $M\left(k_{1}, k_{2}, x\right)$ and $U\left(k_{1}, k_{2}, x\right)$ are independent solutions of Kummer's confluent hypergeometric equation

$$
\frac{d^{2} y}{d x^{2}}+\left(k_{2}-x\right) \frac{d y}{d x}-k_{1} y=0 \text {. }
$$

This equation and its solutions are discussed in detail in Abramowitz and Stegun [1], section (13.1) and Slater [19]. For $a_{\mathrm{s}}=0$, the solution of equation (4.17) is

$$
F(\eta)=\eta^{-n}\left[A I_{m}(\sqrt{\bar{\chi}} \eta)+B K_{m}(\sqrt{\bar{\chi}} \eta)\right],
$$

where $m=|n|$, and $I_{m}(z)$ and $K_{m}(z)$ are modified Bessel functions of the first and second kind (Abramowitz and Stegun [1], section 9.6). The complete solution for $u$ and hence $F_{0}$ is now known and given explicitly by substituting $F(\eta)$ from equations (4.18) or (4.20) into equation (4.15).

In any particular boundary value problem involving the similarity solutions of equations (4.15), (4.18) and (4.20), the boundary conditions are specified on similarity curves $\eta=\eta_{0}=$ constant, and $F\left(\eta_{0}\right)$ (or a linear combination of $F^{\prime}$ and $F$ ) must be chosen accordingly. The parameter $\chi$ appears only in equation (4.17) for $F(\eta)$, and not in the similarity coordinate or boundary condition. Thus $\chi$ can play the role of an eigenvalue (Bluman and Cole [5]).

Case (ii) $\kappa=\kappa_{0}(p) r$

The derivation of the similarity solutions for this case are completely analogous to Case (i). Integration of the trajectory equation (4.3) with the generators (3.21) leads to the similarity variable

$$
\eta(x, t)=\left[x+d_{1}-e_{1}\left(t-t_{5}\right)\right] /\left[\left(t-t_{s}\right)^{2}+a_{s}\right]^{\frac{1}{2}}
$$

and integration of the second trajectory equation (4.4) gives the similarity form 


$$
\begin{aligned}
u= & F(\eta) \exp \left(-\frac{1}{4} \eta^{2}\left(t-t_{s}\right)-\left(1-\frac{1}{2} e_{1}\right)^{2}\left(t-t_{s}\right)-\frac{1}{4} \ln \left[\left(t-t_{s}\right)^{2}+a_{s}\right]\right. \\
& \left.+\eta\left(1-\frac{1}{2} e_{1}\right)\left[\left(t-t_{s}\right)^{2}+a_{s}\right]^{\frac{1}{2}}+c_{1} \int^{t_{-1}} d y /\left(y^{2}+a_{s}\right)\right)
\end{aligned}
$$

where $t_{s}, a_{s}, e_{1}, d_{1}$ and $c_{1}$ are arbitrary parameters. Again the integral in the exponent is given by (4.16). The function $F(\eta)$ in (4.22) satisfies the ordinary differential equation

$$
\frac{d^{2} F}{d \eta^{2}}+\left(\frac{a_{s} \eta^{2}}{4}-c_{1}\right) F(\eta)=0
$$

which is obtained by requiring that the similarity solution satisfy the homogeneous equation of transport (2.6).

The general solution of equation (4.23) for $F(\eta)$ depends on the arbitrary parameter $a_{s}$. For $a_{s} \neq 0$, the solution is

$$
\begin{aligned}
F(\eta)= & \exp \left(-\frac{\left(-a_{s}\right)^{\frac{1}{2}} \eta^{2}}{4}\right)\left[A M\left(\frac{1}{4}+\frac{c_{1}}{\left(-a_{s}\right)^{\frac{1}{2}}}, \frac{1}{2}, \frac{\left(-a_{s}\right)^{\frac{1}{2}} \eta^{2}}{2}\right)\right. \\
& \left.+B U\left(\frac{1}{4}+\frac{c_{1}}{\left(-a_{s}\right)^{\frac{1}{2}}}, \frac{1}{2}, \frac{\left(-a_{s}\right)^{\frac{1}{2}} \eta^{2}}{2}\right)\right]
\end{aligned}
$$

where $M\left(k_{1}, k_{2}, x\right)$ and $U\left(k_{1}, k_{2}, x\right)$ are independent solutions of Kummer's confluent hypergeometric equation (4.19). For $a_{s}=0$ equation (4.23) has solutions in terms of exponential functions:

$$
F(\eta)=A \exp \left(-\sqrt{c_{1}} \eta\right)+B \exp \left(\sqrt{c_{1}} \eta\right) .
$$

Substitution of the expressions (4.24) or (4.25) for $F(\eta)$ into (4.22) gives the general similarity solution for $u$, and hence $F_{0}$, for this case with $\kappa=\kappa_{0}(p) r$.

\section{Solutions for cosmic-ray boundary value problems}

It was noted in the introduction, and section 4 , that the similarity curves $\eta=\eta_{0}=$ constant, can be used as boundary curves in the solution of boundary value problems. In studying the propagation of cosmic-rays in the interplanetary medium, solutions in which we can specify the distribution function $F_{0}(r, p)$ at one fixed heliocentric radius and boundary conditions at another are of particular interest. These solutions are obtained by direct application when the similarity variable $\eta$ is solely a function of $r$, so that $\eta=\eta(r)$. The similarly variables $\eta$ of equations (4.13) and (4.21) are dependent on a set of arbitrary parameters. For particular parameter values and diffusion coefficients we have $\eta=\eta(r)$. Further similarity variables can be obtained by letting one or more of the parameters tend to infinity and 
appropriately rescaling the old similarity variable. The corresponding similarity solutions are obtained by substituting the appropriate parameter values in the similarity solutions of section 4 .

We now obtain three such solutions; two from case (i) with $\kappa=\kappa_{0}(p) r^{b}$, $b \neq 1$, and one from case (ii) for which $\kappa=\kappa_{0}(p) r$. They are given below as Examples 1, 2 and 3.

\section{EXAMPLE 1. $\kappa=\kappa_{c} p^{a} r_{b}, b \neq 1$}

For a diffusion coefficient $\kappa=\kappa_{0}(p) r^{b}, b \neq 1$, the similarity variable is given by equation (4.13), that is

$$
\eta=x /\left[\left(t-t_{s}\right)^{2}+a_{s}\right]^{\frac{1}{2}},
$$

with $x$ and $t$ defined in terms of $r$ and $p$ in equations (2.4) and (2.5).

By choosing $a_{s}=t_{s}=0$ in the similarity variable (5.1) we obtain a particular similarity variable

$$
\eta_{1}=x / t \text {. }
$$

The similarity variable $\eta_{1}$ is solely a function of heliocentric radius $r$ if the diffusion coefficient is of the form

and in this case

$$
\kappa=\kappa_{c} r^{b} p^{3(b-1) / 4},
$$

$$
\begin{aligned}
x & =2\left(r p^{3 / 2}\right)^{(1-b) / 2} /(1-b), \\
t & =2 \kappa_{c} p^{3(1-b) / 4} /[V(b-1)], \\
\eta_{1} & =x / t=\lambda-V r^{(1-b) / 2} / \kappa_{c},
\end{aligned}
$$

are the appropriate expressions for $x, t$ and $\eta_{1}$.

The similarity solution for this case is obtained by putting $a_{s}=t_{s}=0$, in the more general similarity form (4.15) and in the solution (4.20) for $F(\eta)$. We obtain

$$
u=\exp \left(-\eta_{1}^{2} t / 4-\chi / t\right) \eta_{1}^{-n^{-n-1}}\left[A I_{m}\left(\sqrt{\chi} \eta_{1}\right)+B K_{m}\left(\sqrt{\chi} \eta_{1}\right)\right] .
$$

This of course has the form (1.1) or (4.8). The spectrum that can be fitted directly on a boundary at fixed $r$ is of the form $\exp \left(-\frac{1}{4} \eta_{1}^{2} t-\chi / t\right) t^{-n-1}$, with $t$ given in terms of $p$ by $(5.3 c)$. More complex spectra can be fitted by using an appropriately weighted eigenfunction expansion constructed from the general solution (5.4), with the parameter $\chi$ playing the role of an eigenvalue.

EXAMPLE 2. $\kappa=\kappa_{c} p^{a} r^{b}, b \neq 1$

The similarity variable $\eta$ with $b \neq 1$ is given by equation (5.1). Putting $a_{s}=-t_{s}^{2}$, and letting $t_{s} \rightarrow \infty$ we introduce a new similarity variable 


$$
\eta_{2}=\operatorname{limit}_{t_{s} \rightarrow \infty} \frac{t_{s} \eta^{2}}{2}=-\frac{x^{2}}{4 t} .
$$

Thus for a diffusion coefficient of the form

$$
\kappa=\kappa_{c} r^{b}, \quad b \neq 1,
$$

for which

$$
\begin{aligned}
& x=2\left(r p^{3 / 2}\right)^{(1-b) / 2} /(1-b), \\
& t=\kappa_{c} p^{3(1-b) / 2} /[V(b-1)],
\end{aligned}
$$

then the similarity variable

$$
\eta_{2}=-\frac{1}{4} x^{2} / t=V r^{1-b} /\left[\kappa_{c}(1-b)\right],
$$

is solely a function of heliocentric radius.

The similarity solution for the diffusion coefficient case (5.6a) with $\kappa=\kappa_{c} r^{b}, b \neq 1$, is obtained from the similarity form (4.15) and the expression (4.18) for $F(\eta)$ by allowing $\chi$ to be a function of $t_{s}$ such that

$$
\nu=-\operatorname{limit}_{t_{s} \rightarrow \infty} \frac{x}{2 t_{s}},
$$

by putting $a_{s}=-t_{s}^{2}$, letting $t_{s} \rightarrow \infty$, and by scaling out infinite multiplication factors in the solution. It is

$$
u=t^{\nu-(n+1) / 2}\left\{A M\left[\frac{1}{2}(1+n)-\nu, 1+n, \eta_{2}\right]+B U\left[\frac{1}{2}(1+n)-\nu, 1+n, \eta_{2}\right]\right\},
$$

where $A$ and $B$ are arbitrary constants. This solution again has the form (1.1) or (4.8). The spectrum that can be fitted directly on a boundary at fixed $r$ is of the form $t^{\nu-(n+1) / 2}$, which via $(5.6 \mathrm{c})$ is a power law spectrum in momentum $p$. Again, more complex spectra can be fitted by using an appropriately weighted eigenfunction expansion constructed from the general solution (5.8), with $\nu$ playing the role of an eigenvalue.

EXAMPLE 3. $\kappa=\kappa_{0}(p) r$

From equation (4.21) the similarity variable for $\kappa=\kappa_{0}(p) r$ is

$$
\eta=\left[x+d_{1}-e_{1}\left(t-t_{s}\right)\right] /\left[\left(t-t_{s}\right)^{2}+a_{s}\right]^{\frac{1}{2}},
$$

where $x$ and $t$ are defined in general in equations (2.4) and (2.5). We now show that for the special case $\kappa=\kappa_{c} r$ it is possible to introduce a new similarity variable $\eta_{3}$ which is solely a function of $r$. In this particular case of $\kappa=\kappa_{c} r$. we have from (2.4) and (2.5)

$$
x=-\frac{1}{2} \ln \left(2 r^{2} p^{3}\right), \quad t=-3 \kappa_{c} \ln (p) / 2 V .
$$


To obtain $\eta_{3}$ we first introduce a new similarity variable

$$
-\operatorname{limit}_{a \rightarrow \infty} a \eta
$$

with $\eta$ given by (5.9) and $a_{s}=a^{2}$. Making use of equations (5.10) this function is

$$
\frac{1}{2} \ln 2-d_{1}-e_{1} t_{s}+\ln (r)+3 \ln (p) / 2-3 \kappa_{r} e_{1} \ln (p) / 2 V,
$$

and finally putting

$$
e_{1}=V / \kappa_{c}, \quad d_{1}=\frac{1}{2} \ln 2-V t_{s} / \kappa_{c},
$$

we eliminate the dependence on $p$ obtaining for this case

$$
\eta_{3}=\ln (r)
$$

as a new similarity variable dependent only on $r$.

The corresponding similarity solution is obtained by substituting the appropriate parameter values for $a_{s}, e_{1}$ and $d_{1}$ in the similarity form (4.22) with $F(\eta)$ given by equation (4.24), and allowing the parameter $c_{\text {, of equation }}$ (4.22) to be a function of $a_{s}$ such that

$$
\operatorname{limit}_{a_{s} \rightarrow \infty} c_{1} / a_{s}=\nu^{2}
$$

Thus letting $a_{s}=a^{2} \rightarrow \infty$, and setting

$$
c=1-V /\left(2 \kappa_{\mathrm{c}}\right),
$$

the similarity solution becomes

$$
u=\left(A e^{\eta_{3}}+B e^{-m_{3}}\right) \exp \left[\left(\nu^{2}-c^{2}\right)\left(t-t_{s}\right)-c \eta_{3}\right] .
$$

Alternatively in terms of heliocentric radius $r$ and momentum $p$, this solution with $\kappa=\kappa_{c} r$ is

$$
u=F_{0}(r, p)=r^{-c}\left(A r^{\nu}+B r^{-\nu}\right) p^{-3 \kappa_{c}\left(\nu^{2}-c^{2}\right) / 2 \nu} .
$$

Using the similarity solution (5.16) it is possible to fit directly a power law spectrum in momentum $p$ on a boundary at a fixed radius. By appropriate choice of $A$ and $B$, and by superposition of the similarity solutions (5.16), more complex boundary spectra can be fitted.

\section{Solutions without spherical symmetry}

The methods used to obtain the spherically-symmetric solutions can be applied to non-spherically-symmetric cases, and anisotropic diffusion can be included. A particular example relevant to cosmic-ray propagation studies is 
that of a strong radial magnetic field, with diffusion coefficients $\kappa_{\|}$along the field and $\kappa_{\perp}$ perpendicular to the magnetic field. For completeness the equations and solutions are summarised below; full details are given in Webb ([21], Ph.D. thesis).

The diffusion coefficients are assumed to be

$$
\kappa_{\|}=\kappa_{0}(p) r^{b}, \quad \kappa_{\perp} / \kappa_{\|}=e=\text { constant; }
$$

with, as previosuly for $\kappa_{,} \kappa_{0}(p)$ an arbitrary function of momentum $p$. Both $\kappa_{\|}$ and $\kappa_{\perp}$ are assumed to be independent of $\theta$ and $\phi$ with $(r, \theta, \phi)$ spherical polar coordinates centred on the sun with polar axis along the sun's rotation axis, and the solar wind velocity is assumed radial and of constant speed $V$.

The steady-state equation of transport for $F_{0}(r, \theta, \phi, p),(2.1)$ with the diffusion tensor $\kappa$ given by (6.1) can be separated with the same change of variables as previously. Thus if $b \neq 1$, and $\mu=\cos \theta$ we obtain

$$
\begin{gathered}
\frac{\partial^{2} F_{0}}{\partial x^{2}}+\frac{2 n+1}{x} \frac{\partial F_{0}}{\partial x}-\frac{\partial F_{0}}{\partial t}+\frac{e(n+1)^{2}}{x^{2}}\left[\left(1-\mu^{2}\right) \frac{\partial^{2} F_{0}}{\partial \mu^{2}}-2 \mu \frac{\partial F_{0}}{\partial \mu}+\frac{1}{1-\mu^{2}} \frac{\partial^{2} F_{0}}{\partial \phi^{2}}\right] \\
=0,
\end{gathered}
$$

with $x$ and $t$ given in terms of $r$ and $p$ by equations (2.4) and (2.5) respectively, and $n=(b+1) /(1-b)$. If $b=1$ then the separable equation becomes

$$
\frac{\partial^{2} F_{0}}{\partial x^{2}}-2 \frac{\partial F_{0}}{\partial x}-\frac{\partial F_{0}}{\partial t}+e\left[\left(1-\mu^{2}\right) \frac{\partial^{2} F_{0}}{\partial \mu}-2 \mu \frac{\partial F_{0}}{\partial \mu}+\frac{1}{1-\mu^{2}} \frac{\partial^{2} F_{0}}{\partial \phi^{2}}\right]=0,
$$

with $x$ and $t$ given by equations (2.4) and (2.5). Note that putting $\kappa_{\perp} / \kappa_{\|}=e=0$ in (6.2) and (6.3) we obtain the spherically symmetric forms (2.6).

For equation (6.2) the similarity variable $\eta$ is again (4.13) viz.

$$
\eta=x /\left[\left(t-t_{s}\right)^{2}+a_{s}\right]^{\frac{1}{2}},
$$

and the solution for $F_{0}(r, \theta, \phi, p)$ in terms of $\eta, t, \mu$ and $\phi$ is

$$
\begin{aligned}
F_{0}= & \left.\exp \left(-\frac{1}{4} \eta^{2}\left(t-t_{s}\right)-\frac{1}{2}(n+1) \ln \left(t-t_{s}\right)^{2}+a_{s}\right]+\chi \int^{t-t_{s}} \frac{d y}{y^{2}+a_{s}}\right) \\
& \cdot\left(A P_{l}^{m}(\mu)+B Q_{l}^{m}(\mu)\right) e^{ \pm \iota m \phi} F(\eta),
\end{aligned}
$$

in which $P_{l}^{m}(\mu)$ and $Q_{l}^{m}(\mu)$ are associated Legendre functions with $l, m$ integers and $m \leqq l$. The function $F(\eta)$ depends on an arbitrary parameter $a_{s}$. If $a_{s} \neq 0$,

$$
\begin{aligned}
F(\eta)= & \eta^{\zeta-n} \exp \left[-\frac{1}{2} \sqrt{-a_{s}} \eta^{2}\right] \\
& \times\left[A M\left(\frac{1}{2} \chi / \sqrt{ }\left(-a_{s}\right)+\frac{1}{2}(1+\zeta), 1+\zeta, \frac{1}{2} \vee\left(-a_{s}\right) \eta^{2}\right)\right. \\
& \left.+B U\left(\frac{1}{2} \chi / \sqrt{ }\left(-a_{s}\right)+\frac{1}{2}(1+\zeta), 1+\zeta, \frac{1}{2} \sqrt{ }\left(-a_{s}\right) \eta^{2}\right)\right]
\end{aligned}
$$


and if $a_{s}=0$,

where

$$
F(\eta)=\eta^{-n}\left[A I_{\zeta}(\sqrt{\bar{\chi}} \eta)+B K_{\zeta}(\sqrt{\chi} \eta)\right]
$$

$$
\zeta=\left[e l(l+1)+n^{2}\right]^{\frac{1}{2}} .
$$

For equation (6.3), the similarity variable $\eta$ and the similarity solution depend on the diffusion coefficient parameter $e=\kappa_{\perp} / \kappa_{\|}$and on an arbitrary parameter $\Delta$ and we have three forms of solution.

(a) If $\kappa_{\perp} / \kappa_{\|}=e \neq 0, \Delta \neq 0$, the similarity variable is

$$
\eta=x-\frac{1}{2} \gamma\left(t-t_{\mathrm{s}}\right)^{2} / \Delta,
$$

and the corresponding solution for $F_{0}(r, \theta, \phi, p)$ is

$$
\begin{aligned}
F_{0}= & \exp \left[-\gamma^{2}\left(t-t_{5}\right)^{3} / 12 \Delta^{2}+\frac{1}{2} \gamma\left(t-t_{5}\right)^{2} / \Delta+\gamma\left(a-\frac{1}{2} \eta\right)\left(t-t_{s}\right) / \Delta\right] \\
& \cdot e^{\eta} z^{1 / 3}\left[A J_{1 / 3}(z)+B J_{-1 / 3}(z)\right] \\
& \cdot\left[C P_{\imath}^{m}(\mu)+D Q_{1}^{m}(\mu)\right] e^{ \pm m \phi}
\end{aligned}
$$

where

$$
z=\frac{4 \Delta}{3 \gamma}\left[\frac{\gamma \eta}{2 \Delta}-\frac{\gamma a}{\Delta}-1-e l(l+1)\right]^{3 / 2} .
$$

(b) If $\kappa_{\perp} / \kappa_{\|}=e \neq 0$ and $\Delta=0$, the similarity variable is

$$
\eta=t-t_{s}
$$

and the solution is

$$
\begin{aligned}
F_{0}= & \frac{1}{\sqrt{ }\left(t-t_{s}\right)} \exp \left[-\frac{x^{2}}{4\left(t-t_{s}\right)}+\frac{\left(t-t_{s}+a\right) x}{4}-\frac{a^{2}}{\left(t-t_{s}\right)}-\left(t-t_{s}\right)[1+e l(l+1)]\right] \\
& \cdot\left[C P_{l}^{m}(\mu)+D Q_{l}^{m}(\mu)\right] e^{ \pm m \phi} .
\end{aligned}
$$

(c) For the special case of (6.3) with $\kappa_{\perp} / \kappa_{\|}=e=0$, the solution is

$$
F_{0}=u(x, t) h(\theta, \phi),
$$

where $u(x, t)$ is the spherically symmetric solution for $\kappa=\kappa_{0}(p) r$, given in equations (4.22), (4.24) and (4.25), and $h(\theta, \phi)$ is an arbitrary function of $\theta$ and $\phi$.

\section{Concluding remarks}

The main aim of this paper has been to construct similarity solutions of the steady-state equation of transport for cosmic-rays in the interplanetary 
region with a diffusion tensor proportional to $\kappa_{0}(p) r^{b}$, and a radial solar-wind velocity, of constant speed $V$. The similarity solutions obtained are (4.15), $(4.22),(6.4),(6.9)$ and $(6.12)$; they are new results containing many new special cases.

Complete details of the derivation of the spherically symmetric similarity solutions were given (section 4). The group methods used have also been applied to derive solutions for non-spherically-symmetric models, and examples of non-spherically symmetric solutions for a diffusion tensor symmetric about the radial direction are listed (section 6). The detailed derivations of these latter solutions are not presented here, but are available in Webb ([21] Ph.D. thesis).

Although not developed here, the spherically symmetric solutions can be used to obtain solutions in which the distribution function is known on a boundary at infinity. These are called galactic-spectrum solutions. One example of these solutions has been obtained by Fisk and Axford [10], using particular cases of the more general separation variables deduced here.

The special solutions obtained in section 5 for which the similarity variable $\eta$ is solely a function of $r$ are of particular interest in cosmic-ray studies. This is because it is possible to use them when the distribution function $F_{0}(r, p)$ is specified at one fixed radial distance $r_{b}$ (say) and appropriate boundary conditions are specified at another. Note that with $\eta=\eta(r)$, the boundary spectrum $F_{0}\left(r_{b}, p\right)$ must have a $p$ dependence dictated by the solution. However, this limitation may be overcome by using a suitable superposition of the solutions obtained here (see last paragraph).

The solutions in all cases were obtained for infinitesimal generators $X, T$, $U$ of the form

$$
X=X(x, t), \quad T=T(t), \quad U=u f(x, t)+g(x, t),
$$

(equations 4.2) for the case $g=0, \gamma \neq 0$. Further solutions could be obtained with $g(x, t)$ a non-trivial solution of the homogeneous equation of transport (2.1). The similarity solutions were constructed from the "classical" subgroup of the transport equation. It is possible that more general solutions could be obtained by using the "non-classical" procedure of Bluman and Cole [5].

The generators were obtained both for the equation of transport with no sources [(3.18), (3.21] and for the case with a monoenergetic source [(3.20), (3.22)]. The subgroup admitted by the equation of transport with a monoenergetic source term can be used to derive the monoenergetic-source solution given in Webb and Gleeson [22] without proof and by Toptygin [20] who derived it without group methods.

The derivation of the monoenergetic-source solution will be given in a 
further paper. Also in preparation is a paper showing how, using the present reults, solutions may be obtained for the general boundary value problem in which $F_{0}(r, p)$ may have any specified form on a boundary at $r=r_{b}$.

\section{Acknowledgements}

The author is indebted to E. D. Fackerell for an introduction to the use of Lie groups in solving partial differential equations, and to L. J. Gleeson for a critical reading and discussion of the manuscript.

\section{References}

[1] M. Abramowitz and I. A. Stegun, Handbook of Mathematical Functions, Dover, New York (1965).

[2] G. W. Bluman, Construction of Solutions to Partial Differential Equations by the use of Lie Groups, Ph.D. Thesis, California Institute of Technology, (1967).

[3] G. W. Bluman, 'Similarity solutions of the one-dimensional Fokker-Planck equation', Int. J. Non-Lin. Mech. 6, (1971), 143.

[4] G. W. Bluman, Applications of the general similarity solution of the heat equation to boundary value problems', Quart. J. Appl. Math., 31, (1974), 403.

[5] G. W. Bluman and J. D. Cole, 'The general similarity solution of the heat equation', J. Math. and Mechanics, 18 (1969), 1025.

[6] G W. Bluman and J. D Cole, Similarity Methods for Differential Equatıons, (A.M.S. publications 13), Springer-Verlag, New York (1974).

[7] A. Z. Dolginov and I. N. Toptygin, 'Diffusion of cosmic particles in the interplanetary medium', Geomag. and Aeron., 7 (1967), 785.

[8] A. Z. Dolginov and I. N. Toptygin, 'Cosmic-rays in the interplanetary magnetic fields', Icarus, 8 (1968), 54.

[9] L. A. Fisk, Behaviour of Cosmic-Rays in the Interplanetary Medium, Ph.D. Thesis, University of California. San Diego, (1969).

[10] L. A. Fisk and W. I. Axford, 'Solar modulation of galactic cosmic-rays, l', J. Geophys Res., 74 (1969), 4973.

[11] L. J. Gleeson and W. I. Axford, 'Cosmic-rays in the interplanetary medium', Astrophys. J. Lett., 149 (1967), L115.

[12] L. J. Gleeson and G. M. Webb, 'Modulation and spectral redistribution of galactic cosmic-rays', Proc. 14th Int. Conf. on Cosmic-Rays, Munich, 3 (1975), 893.

[13] J. R. Jokipu and E. N. Parker, 'On the convection, diffusion and adiabatic deceleration of cosmic-rays in the solar wind", Astrophys. J, 172 (1970), 319.

[14] S. Lie, 'Über dıe Integratıon durch bestımmte Integrale Von eıner Klasse lınearer partieller Differentialgleichungen', Arch Math., VI, (1881), 328.

[15] E. A Muller and K Matschat, Über das Auffinden von Ähnlıchkeitslosungen partieller Differentialgleichungssysteme, mit Anwendung auf Probleme der Stromungphysik, Mizellaneen der Angewandten Mechanık', Berlin, (1962), 190.

[16] L. V. Ovsjannikov, Gruppovye svoystva differentisialny uravneni, Novosibirsk (1962) (Group Properties of Differential Equations, translated by G. W. Bluman, 1967).

[17] E. N. Parker, 'The passage of energetic charged particles through interplanetary space', Planet. Space Sct., 13 (1965), 9.

[18] E. N. Parker, "The effect of adiabatic deceleration on the cosmic-ray spectrum in the solar system', Planet. Space Scı., 14 (1966), 371. 
[19] L. J. Slater, Confluent Hypergeometric Functions, Cambridge University Press, London (1960).

[20] 1. N. Toptygin, 'Direct and inverse problem of cosmic-ray propagation in interplanetary space', Geomag. and Aeron., 13 (1973), 181.

[21] G. M. Webb, Steady-state cosmic-ray propagation in interplanetary space, Ph.D. Thesis, University of Tasmania, Hobart, (1976).

[22] G. M. Webb and L. J. Gleeson, 'Monoenergetic-source solutions of the steady-state cosmic-ray equation of transport', Proc. 13th Int. Conf. on Cosmic-Rays, Denver, (University of Denver), Conf. Papers, 5 (1973), 3253.

[23] G. M. Webb and L. J. Gleeson, 'Solutions of the cosmic-ray equation of transport', Proc. Astron. Soc. Aust., Vol. 2, No. 5, (1974), 299.

Department of Mathematics, Monash University,

Clayton, Vic. 3168,

Australia 